\title{
Stabilization and Controller Design of 2D Discrete Switched Systems with State Delays under Asynchronous Switching
}

\author{
Shipei Huang, ${ }^{1}$ Zhengrong Xiang, ${ }^{1}$ and Hamid Reza Karimi ${ }^{2}$ \\ ${ }^{1}$ School of Automation, Nanjing University of Science and Technology, Nanjing 210094, China \\ ${ }^{2}$ Department of Engineering, Faculty of Engineering and Science, University of Agder, 4898 Grimstad, Norway \\ Correspondence should be addressed to Zhengrong Xiang; xiangzr@mail.njust.edu.cn
}

Received 22 February 2013; Accepted 14 April 2013

Academic Editor: Jun $\mathrm{Hu}$

Copyright (c) 2013 Shipei Huang et al. This is an open access article distributed under the Creative Commons Attribution License, which permits unrestricted use, distribution, and reproduction in any medium, provided the original work is properly cited.

\begin{abstract}
This paper is concerned with the problem of robust stabilization for a class of uncertain two-dimensional (2D) discrete switched systems with state delays under asynchronous switching. The asynchronous switching here means that the switching instants of the controller experience delays with respect to those of the system. The parameter uncertainties are assumed to be norm-bounded. A state feedback controller is proposed to guarantee the exponential stability. The dwell time approach is utilized for the stability analysis and controller design. A numerical example is given to illustrate the effectiveness of the proposed method.
\end{abstract}

\section{Introduction}

Two-dimensional (2D) systems have attracted considerable attention for several decades due to their numerous applications in many areas, such as multidimensional digital filtering, linear image processing, signal processing and process control [1-3]. It is well known that 2D systems can be represented by different models such as Roesser model, FornasiniMarchesini model, and Attasi model. The issues of stability analysis and control synthesis of these systems have been studied in [4-8]. Considering that time delays frequently occur in practical systems and are often the source of instability, many authors have devoted their energies to studying time-delay systems. Recently, many results on delay systems have been reported in the literature. For example, the delayfractional approach has been utilized to deal with discrete time-delay systems in [9-11]. The stability of 2D discrete systems with state delays has been investigated in [12-16].

On the other hand, because of their wide applications in many fields, such as mechanical systems, automotive industry, aircraft and air traffic control, and switched power converters, switched systems have also received considerable attention during the past few decades. A switched system is a hybrid system which consists of a finite number of continuous-time or discrete-time subsystems and a switching signal specifying the switch between these subsystems. The stability and stabilization problems have been extensively studied in [17-25].

In many modelling problems of physical processes, a $2 \mathrm{D}$ switching representation is needed. One can cite a $2 \mathrm{D}$ physically based model for advanced power bipolar devices [26] and heat flux switching, and modulating in a thermal transistor [27]. This class of systems can correspond to 2D state space or 2D time space switched systems. Recently, there are a few reports on 2D switched systems. Benzaouia et al. firstly studied the stabilization problem of $2 \mathrm{D}$ discrete switched systems with arbitrary switching sequences in [28, 29]. By using the common Lyapunov function method and multiple Lyapunov functions method, two different sufficient conditions for the existence of state feedback controllers were proposed. In [30], the authors first extended the concept of average dwell time to $2 \mathrm{D}$ switched systems and designed a switching signal to guarantee the exponential stability of delay-free 2D switched systems. It should be pointed out that a very common assumption in [30] is that the controllers are switched synchronously with the switching of system modes, which is quite unpractical. As stated in [31, 32], there inevitably exists asynchronous switching in actual operation that is, the switching instants of the controllers exceed or lag behind those of system modes. Thus, it is necessary to 
consider asynchronous switching for efficient control design. Some results on the control synthesis for switched systems under asynchronous switching have been proposed in [3336]. However, to the best of our knowledge, the stabilization problem for 2D switched systems under asynchronous switching has not been yet investigated to date, especially for 2D switched systems with state delays, which motivates our present study.

In this paper, we are interested in designing a stabilizing controller for 2D discrete switched delayed systems represented by a model of Roesser type under asynchronous switching such that the corresponding closed-loop systems are exponentially stable. The dwell time approach is utilized for the stability analysis and controller design. The main contributions of this paper can be summarized as follows: (i) the asynchronous stabilization problem is for the first time addressed in the paper; (ii) an exponential stability criterion is established for 2D switched systems with state delays; and (iii) an asynchronous switching controller design scheme is proposed to guarantee the exponential stability of the resulting closed-loop system.

This paper is organized as follows. In Section 2, problem formulation and some necessary lemmas are given. In Section 3, based on the dwell time approach, stability and stabilization for $2 \mathrm{D}$ discrete switched systems with state delays are addressed. Then, a sufficient condition for the existence of a stabilizing controller for such 2D discrete switched systems under asynchronous switching is derived in terms of a set of matrix inequalities. A numerical example is provided to illustrate the effectiveness of the proposed approach in Section 4. Concluding remarks are given in Section 5.

Notations. Throughout this paper, the superscript " $T$ " denotes the transpose, and the notation $X \geq Y(X>Y)$ means that the matrix $X-Y$ is positive semidefinite (positive definite, resp.). $\|\cdot\|$ denotes the Euclidean norm. I represents the identity matrix with an appropriate dimension. $I_{h}$ is the identity matrix with $n_{1}$ dimension and $I_{v}$ is the identity matrix with $n_{2}$ dimension. $\operatorname{diag}\left\{a_{i}\right\}$ denotes the diagonal matrix with the diagonal elements $a_{i}$, and $i=1,2, \ldots, n$. $X^{-1}$ denotes the inverse of $X$. The asterisk $*$ in a matrix is used to denote the term that is induced by symmetry. The set of all nonnegative integers is represented by $Z_{+}$.

\section{Problem Formulation and Preliminaries}

Consider the following uncertain 2D discrete switched systems with state delays:

$$
\begin{aligned}
& {\left[\begin{array}{l}
x^{h}(i+1, j) \\
x^{v}(i, j+1)
\end{array}\right]} \\
& =\widehat{A}^{\sigma(i, j)}\left[\begin{array}{l}
x^{h}(i, j) \\
x^{v}(i, j)
\end{array}\right]+\widehat{A}_{d}^{\sigma(i, j)}\left[\begin{array}{l}
x^{h}\left(i-d_{h}, j\right) \\
x^{v}\left(i, j-d_{v}\right)
\end{array}\right] \\
& +\widehat{B}^{\sigma(i, j)} u(i, j),
\end{aligned}
$$

where $x^{h}(i, j) \in R^{n_{1}}$ and $x^{v}(i, j) \in R^{n_{2}}$ are the horizontal state and the vertical state, respectively, $x(i, j)$ is the whole state in
$R^{n}$ with $n=n_{1}+n_{2}$, and $u(i, j) \in R^{m}$ is the control input. $i$ and $j$ are integers in $Z_{+} \cdot \sigma(i, j): Z_{+} \times Z_{+} \rightarrow \underline{N}=$ $\{1,2, \ldots, N\}$ is the switching signal. $N$ is the number of subsystems, and $\sigma(i, j)=k$ means that the $k$ th subsystem is activated. $d_{h}$ and $d_{v}$ are constant delays along horizontal and vertical directions, respectively. $\widehat{A}^{k}$ and $\widehat{A}_{d}^{k}(k \in \underline{N})$ are uncertain real-valued matrices with appropriate dimensions and are assumed to be of the form

$$
\begin{aligned}
& \widehat{A}^{k}=A^{k}+H^{k} F^{k}(i, j) E_{1}^{k}, \\
& \widehat{A}_{d}^{k}=A_{d}^{k}+H^{k} F^{k}(i, j) E_{2}^{k}, \\
& \widehat{B}^{k}=B^{k}+H^{k} F^{k}(i, j) E_{3}^{k},
\end{aligned}
$$

with

$$
\begin{aligned}
& A^{k}=\left[\begin{array}{ll}
A_{11}^{k} & A_{12}^{k} \\
A_{21}^{k} & A_{22}^{k}
\end{array}\right], \quad A_{d}^{k}=\left[\begin{array}{ll}
A_{d 11}^{k} & A_{d 12}^{k} \\
A_{d 21}^{k} & A_{d 22}^{k}
\end{array}\right], \\
& H^{k}=\left[\begin{array}{c}
H_{1}^{k} \\
H_{2}^{k}
\end{array}\right], \quad E_{1}^{k}=\left[\begin{array}{c}
E_{11}^{k} \\
E_{12}^{k}
\end{array}\right] \text {, } \\
& E_{2}^{k}=\left[\begin{array}{c}
E_{21}^{k} \\
E_{22}^{k}
\end{array}\right], \quad E_{3}^{k}=\left[\begin{array}{c}
E_{31}^{k} \\
E_{32}^{k}
\end{array}\right] \text {, }
\end{aligned}
$$

where matrices $A_{11}^{k} \in R^{n_{1} \times n_{1}}, A_{12}^{k} \in R^{n_{1} \times n_{2}}, A_{21}^{k} \in R^{n_{2} \times n_{1}}$, $A_{22}^{k} \in R^{n_{2} \times n_{2}}, A_{d 11}^{k} \in R^{n_{1} \times n_{1}}, A_{d 12}^{k} \in R^{n_{1} \times n_{2}}, A_{d 21}^{k} \in R^{n_{2} \times n_{1}}$, $A_{d 22}^{k} \in R^{n_{2} \times n_{2}}, H_{1}^{k}, H_{2}^{k}, E_{11}^{k}, E_{12}^{k}, E_{21}^{k}, E_{22}^{k}, E_{31}^{k}, E_{32}^{k}$ are constant matrices. $F^{k}(i, j)(k \in \underline{N})$ is an unknown matrix representing parameter uncertainty and satisfies

$$
F^{k T}(i, j) F^{k}(i, j) \leq I \text {. }
$$

The boundary conditions are given by

$$
\begin{gathered}
x^{h}(i, j)=h_{i j}, \quad \forall 0 \leq j \leq z_{1},-d_{h} \leq i \leq 0, \\
x^{h}(i, j)=0, \quad \forall j>z_{1}, \quad-d_{h} \leq i \leq 0, \\
x^{v}(i, j)=v_{i j}, \quad \forall 0 \leq i \leq z_{2},-d_{v} \leq j \leq 0, \\
x^{v}(i, j)=0, \quad \forall i>z_{2},-d_{v} \leq j \leq 0,
\end{gathered}
$$

where $z_{1}<\infty$ and $z_{2}<\infty$ are positive integers, and $h_{i j}$ and $v_{i j}$ are given vectors.

In this paper, it is assumed that (1) at each time only one subsystem is active; (2) the switching signal is not known a priori, but its value is available at each sampling period; (3) the switch occurs only at each sampling point of $i$ or $j$. The switching sequence can be described as follows:

$$
\begin{aligned}
& \left(\left(i_{0}, j_{0}\right), \sigma\left(i_{0}, j_{0}\right)\right),\left(\left(i_{1}, j_{1}\right), \sigma\left(i_{1}, j_{1}\right)\right), \ldots, \\
& \left(\left(i_{\kappa}, j_{\kappa}\right), \sigma\left(i_{\kappa}, j_{\kappa}\right)\right), \ldots,
\end{aligned}
$$

where $\left(i_{\kappa}, j_{\kappa}\right)$ denotes the $\kappa$ th switching instant. It should be noted that the value of $\sigma(i, j)$ only depends on $i+j$ (see [29, 30]). 
However, in actual operation, there inevitably exists asynchronous switching between the controller and the system. Without loss of generality, we only consider the case where the switching instants of the controller experience delays with respect to those of the system. Let $\sigma^{\prime}(i, j)$ denote the switching signal of the controller. Denoting $m_{\kappa}=i_{\kappa}+j_{\kappa}, \Delta m_{\kappa}=\Delta i_{\kappa}+$ $\Delta j_{\mathcal{K}}, \kappa=1,2, \ldots$, then the switching points of the controller can be described as

$$
\left(i_{0}, j_{0}\right),\left(i_{1}+\Delta i_{1}, j_{1}+\Delta j_{1}\right), \ldots,\left(i_{\kappa}+\Delta i_{\kappa}, j_{\kappa}+\Delta j_{\kappa}\right), \ldots,
$$

where $\Delta i_{\kappa}$ and $\Delta j_{\mathcal{K}}$ represent the delayed period along horizontal and vertical directions, respectively. $\Delta m_{\kappa}<\inf \left(m_{\kappa+1}-\right.$ $m_{\kappa}$ ) is said to be the mismatched period.

Remark 1. Similar to the one-dimensional switched system case [33-36], the mismatched period $\Delta m_{\kappa}<\inf \left(m_{\kappa+1}-m_{\kappa}\right)$ guarantees that there always exists a period in which the controller and the system operate synchronously. This period is said to be the matched period in the later section.

Remark 2. If there is only one subsystem in system (1), it will degenerate to the following $2 \mathrm{D}$ system in Roesser model with state delays [12]:

$$
\left[\begin{array}{l}
x^{h}(i+1, j) \\
x^{v}(i, j+1)
\end{array}\right]=\widehat{A}\left[\begin{array}{l}
x^{h}(i, j) \\
x^{v}(i, j)
\end{array}\right]+\widehat{A}_{d}\left[\begin{array}{l}
x^{h}\left(i-d_{h}, j\right) \\
x^{v}\left(i, j-d_{v}\right)
\end{array}\right],
$$

Definition 3. System (1) is said to be exponentially stable under $\sigma(i, j)$ if, for a given $z \geq 0$, there exist positive constants $c$ and $\eta$, such that

$$
\sum_{i+j=D}\|x(i, j)\|^{2} \leq \eta e^{-c(D-z)} \sum_{i+j=z}\|x(i, j)\|_{C}^{2}
$$

holds for all $D \geq z$, where

$$
\begin{aligned}
& \sum_{i+j=z}\|x(i, j)\|_{C}^{2} \\
& \triangleq \sup _{\substack{-d_{h} \leq \theta_{h} \leq 0,-d_{v} \leq \theta_{v} \leq 0}} \sum_{i+j=z}\left(\left\|x^{h}\left(i-\theta_{h}, j\right)\right\|^{2}+\left\|x^{v}\left(i, j-\theta_{v}\right)\right\|^{2}\right) .
\end{aligned}
$$

Remark 4. From Definition 3, it is easy to see that when $z$ is given, $\sum_{i+j=z}\|x(i, j)\|_{C}^{2}$ will be bounded and $\sum_{i+j=D}\|x(i, j)\|^{2}$ will tend to be zero exponentially as $D$ goes to infinity, which implies that $\|x(i, j)\|$ tends to be zero.

Definition 5. Let $\left(i_{\kappa}, j_{\kappa}\right)$ denote the $\kappa$ th switching point and $\left(i_{\kappa+1}, j_{\kappa+1}\right)$ denote the $(\kappa+1)$ th switching point. Denote $m_{\kappa}=$ $i_{\kappa}+j_{\mathcal{K}}, m_{\kappa+1}=i_{\kappa+1}+j_{\kappa+1}, \tau=\inf \left(m_{\kappa+1}-m_{\kappa}\right)$, then $\tau$ is called the dwell time.

Definition 6 (see [30]). For any $i+j=D \geq z=i_{z}+j_{z}$, let $N_{\sigma(i, j)}(z, D)$ denote the switching number of $\sigma(i, j)$ on the interval $[z, D)$. If

$$
N_{\sigma(i, j)}(z, D) \leq N_{0}+\frac{D-z}{\tau_{a}}
$$

holds for given $N_{0} \geq 0$ and $\tau_{a} \geq 0$, then the constant $\tau_{a}$ is called the average dwell time and $N_{0}$ is the chatter bound.

Lemma 7 (see [37]). For a given matrix $S=\left[\begin{array}{ll}S_{11} & S_{12} \\ S_{12}^{T} & S_{22}\end{array}\right]$, where $S_{11}$ and $S_{22}$ are square matrices, the following conditions are equivalent:

(i) $S<0$,

(ii) $S_{11}<0, S_{22}-S_{12}^{T} S_{11}^{-1} S_{12}<0$,

(iii) $S_{22}<0, S_{11}-S_{12} S_{22}^{-1} S_{12}^{T}<0$.

Lemma 8 (see [38]). Let $U, V, W$, and $X$ be real matrices of appropriate dimensions with $X$ satisfying $X=X^{T}$, then for all $V^{T} V \leq I$,

$$
X+U V W+W^{T} V^{T} U^{T}<0,
$$

if and only if there exists a scalar $\varepsilon$ such that

$$
X+\varepsilon U U^{T}+\varepsilon^{-1} W^{T} W<0 .
$$

\section{Main results}

3.1. Stability Analysis. In this section, we first focus on the stability analysis for system (8).

Lemma 9. Consider system (8) with the boundary conditions (5), suppose that there exists a $C^{1}$ function $V: R^{n} \rightarrow R$. For a given positive constant $\alpha$, if there exist positive definite symmetric matrices $P=\operatorname{diag}\left\{P_{h}, P_{v}\right\}$ and $Q=\operatorname{diag}\left\{Q_{h}, Q_{v}\right\}$ with appropriate dimensions, such that the following inequality holds:

$$
\left[\begin{array}{ccc}
Q-\alpha P & 0 & \widehat{A}^{T} P \\
* & -\Lambda_{1} Q & \widehat{A}_{d}^{T} P \\
* & * & -P
\end{array}\right]<0
$$

where $\Lambda_{1}=\operatorname{diag}\left\{\alpha^{d_{h}} I_{h}, \alpha^{d_{v}} I_{v}\right\}$, then along the trajectory of systems (8), the following inequality holds for any $D \geq D^{\prime}$ :

$$
\sum_{i+j=D} V(x(i, j))<\alpha^{D-D^{\prime}} \sum_{i+j=D^{\prime}} V(x(i, j)),
$$

where $D^{\prime} \geq z$ and $z=\max \left\{z_{1}, z_{2}\right\}$.

Proof. See appendix for the detailed proof, it is omitted here.

Remark 10. Lemma 9 provides a method for the estimation of the $C^{1}$ function $V$ which will be used to design the controller for system (1) under asynchronous switching. It is worth pointing out that when $0<\alpha<1$, (15) presents the decay estimation of the $C^{1}$ function $V$, and when $\alpha>1$, (15) shows the growth estimation of the $C^{1}$ function $V$.

Remark 11. It is noted that the block diagonal matrices $P$ and $Q$ are often chosen as the matrices for Lyapunov functional analysis of $2 \mathrm{D}$ systems by the Roesser model in the existing literature (see, e.g., $[12,13,15])$. This is because $2 \mathrm{D}$ systems in Roesser model may be unstable when the block diagonal matrices are not chosen, which has been shown in the literature $[4,5]$. 
3.2. Controller Design. Consider system (1), under the following asynchronous switching controller:

$$
u(i, j)=K^{\sigma^{\prime}(i, j)} x(i, j), \quad K^{\sigma^{\prime}(i, j)}=\left[\begin{array}{ll}
K_{1}^{\sigma^{\prime}(i, j)} & K_{2}^{\sigma^{\prime}(i, j)}
\end{array}\right],
$$

where $K_{1}^{\sigma^{\prime}(i, j)} \in R^{m \times n_{1}}$ and $K_{2}^{\sigma^{\prime}(i, j)} \in R^{m \times n_{2}}$, the corresponding closed-loop system is given by

$$
\begin{aligned}
& {\left[\begin{array}{l}
x^{h}(i+1, j) \\
x^{v}(i, j+1)
\end{array}\right]} \\
& =\left(\widehat{A}^{\sigma(i, j)}+\widehat{B}^{\sigma(i, j)} K^{\sigma^{\prime}(i, j)}\right)\left[\begin{array}{l}
x^{h}(i, j) \\
x^{v}(i, j)
\end{array}\right] \\
& +\widehat{A}_{d}^{\sigma(i, j)}\left[\begin{array}{l}
x^{h}\left(i-d_{h}, j\right) \\
x^{v}\left(i, j-d_{v}\right)
\end{array}\right] .
\end{aligned}
$$

Without loss of generality, we denote $\sigma\left(i_{\kappa}, j_{\kappa}\right)=k \in \underline{N}$ and $\sigma\left(i_{\kappa+1}, j_{\kappa+1}\right)=l \in \underline{N}$, then due to the existence of asynchronous switching, we can obtain from (7) that

$$
\begin{gathered}
\sigma^{\prime}\left(i_{\kappa}+\Delta i_{\kappa}, j_{\mathcal{K}}+\Delta j_{\mathcal{K}}\right)=k, \\
\sigma^{\prime}\left(i_{\kappa+1}+\Delta i_{\kappa+1}, j_{\kappa+1}+\Delta j_{\kappa+1}\right)=l .
\end{gathered}
$$

In many actual applications, it is always difficult to verify each asynchronous period in advance, but the maximal asynchronous period can be easily predicted offline. Let $\Delta=$ $\max _{\kappa=1,2, \ldots}\left(\Delta m_{\kappa}\right)$ denote the maximal asynchronous period, then we can get the following result.

Theorem 12. Consider system (1), for given positive constants $\alpha<1$ and $\beta>1$, if there exist positive definite symmetric matrices $X^{k}=\operatorname{diag}\left\{X_{h}^{k}, X_{v}^{k}\right\}, Y^{k}=\operatorname{diag}\left\{Y_{h}^{k}, Y_{v}^{k}\right\}, X^{k l}=$ $\operatorname{diag}\left\{X_{h}^{k l}, X_{v}^{k l}\right\}, Y^{k l}=\operatorname{diag}\left\{Y_{h}^{k l}, Y_{v}^{k l}\right\}$ and $W^{k}$ with appropriate dimensions, and positive scalars $\varepsilon_{k}$ and $\varepsilon_{k l}$ such that, for $k, l \in$ $\underline{N}, k \neq l$, the following inequalities hold,

$$
\left[\begin{array}{ccccc}
-\alpha X^{k} & 0 & \left(A^{k} X^{k}+B^{k} W^{k}\right)^{T} & X^{k} & \left(E_{1}^{k} X^{k}+E_{3}^{k} W^{k}\right)^{T} \\
* & -\Lambda_{1} Y^{k} & \left(A_{d}^{k} Y^{k}\right)^{T} & 0 & \left(E_{2}^{k} Y^{k}\right)^{T} \\
* & * & -X^{k}+\varepsilon_{k} H^{k} H^{k T} & 0 & 0 \\
* & * & * & -Y^{k} & 0 \\
* & * & * & * & -\varepsilon_{k} I
\end{array}\right]<0,
$$

$$
\left[\begin{array}{ccccc}
-\beta X^{k l} & 0 & \left(A^{l} X^{k l}+B^{l} K^{k} X^{k l}\right)^{T} & X^{k l} & \left(E_{1}^{l} X^{k l}+E_{3}^{l} K^{k} X^{k l}\right)^{T} \\
* & -\Lambda_{2} Y^{k l} & \left(A_{d}^{l} Y^{k l}\right)^{T} & 0 & \left(E_{2}^{l} Y^{k l}\right)^{T} \\
* & * & -X^{k l}+\varepsilon_{k l} H^{l} H^{l T} & 0 & 0 \\
* & * & * & -Y^{k l} & 0 \\
* & * & * & * & -\varepsilon_{k l} I
\end{array}\right]<0
$$

where $\Lambda_{2}=\operatorname{diag}\left\{\beta^{d_{h}} I_{h}, \beta^{d_{v}} I_{v}\right\}$, then under the following switching controller:

$$
u(i, j)=K^{\sigma^{\prime}(i, j)} x(i, j), \quad K^{k}=W^{k}\left(X^{k}\right)^{-1},
$$

and the following average dwell time scheme:

$$
\tau_{a}>\tau_{a}^{*}=\frac{\Delta(\ln \beta-\ln \alpha)+\ln \left(\mu_{1} \mu_{2}\right)}{-\ln \alpha},
$$

the resulting closed-loop system (17) is exponentially stable, where $\mu=(\alpha / \beta)^{\bar{d}}, \bar{d}=\max \left\{d_{h}, d_{v}\right\}$, and $\mu_{1} \mu_{2} \mu \geq 1$ satisfies

$$
\begin{array}{lc}
X_{l}^{-1} \leq \mu_{1} X_{k l}^{-1}, & X_{k l}^{-1} \leq \mu_{2} X_{k}^{-1}, \\
Y_{l}^{-1} \leq \mu_{1} Y_{k l}^{-1}, & Y_{k l}^{-1} \leq \mu_{2} \mu Y_{k}^{-1} .
\end{array}
$$

Proof. See the appendix.

Remark 13. In Theorem 12, we propose a sufficient condition for the existence of a state feedback controller such that the resulting closed-loop system (17) is exponentially stable. It is worth noting that this condition is obtained by using the average dwell time approach. Here, $\alpha$ plays a key role in controlling the rate of decaying of the system in the matched period, and $\beta$ plays a key role in controlling the rate of increasing of the system in the mismatched period. From (22), we know that the value of $\tau_{a}^{*}$ is proportional to the value of $\Delta$, which also means that it needs much larger dwell time to guarantee the stability of the considered system when there exists much larger asynchronous period.

Remark 14. It is noticed that (19) and (20) are mutually dependent. Therefore, we can firstly solve (19) to obtain the solution of matrices $X^{k}, Y^{k}$, and $W^{k}$. Then, (20) can be transformed into the LMI by substituting $K^{k}=W^{k}\left(X^{k}\right)^{-1}$ into it. By adjusting the parameters $\alpha$ and $\beta$, we can find a feasible solution of $X^{k}, Y^{k}, W^{k}, X^{k l}$, and $Y^{k l}$ such that (19) and (20) hold.

The procedure of the controller design for system (1) can be given as follows.

Step 1. Given system matrices and positive scalar $0<\alpha<$ 1 , and by solving LMI (19), we can get the feasible solution of matrices $X^{k}, Y^{k}$, and $W^{k}$ and positive scalar $\varepsilon_{k}$ then, the controller can be obtained from (21). 
Step 2. Substituting $K^{k}$ into (20), we can find the feasible solution of $X^{k l}, Y^{k l}$, and $\varepsilon_{k l}$ such that (20) holds by adjusting the parameter $\beta$.

Step 3. From (23), we can obtain $\mu_{1}$ and $\mu_{2}$ satisfying $\mu_{1} \mu_{2} \mu \geq$ 1.

Step 4. Taking the value of $\Delta$, we can compute the value of $\tau_{a}^{*}$ by $(22)$.

Remark 15. From the procedure above, it can be seen that the proposed method is feasible. We can find the desire controller and switching signal according to the procedure. However, we would like to point out that there still exists the conservatism to some extent for this method because (19) and (20) are mutually dependent, which brings about the increase of the complex computation. The result can be improved by adopting the method presented in $[31,32]$.

When $\widehat{A}_{d}^{\sigma(i, j)}=0$, system (17) degenerates to the following delay-free system:

$$
\left[\begin{array}{l}
x^{h}(i+1, j) \\
x^{v}(i, j+1)
\end{array}\right]=\left(\widehat{A}^{\sigma(i, j)}+\widehat{B}^{\sigma(i, j)} K^{\sigma^{\prime}(i, j)}\right)\left[\begin{array}{l}
x^{h}(i, j) \\
x^{v}(i, j)
\end{array}\right] .
$$

Then, we can get the following result.

Corollary 16. Consider system (1) with $\widehat{A}_{d}^{\sigma(i, j)}=0$, for given positive constants $\alpha<1$ and $\beta>1$, if there exist positive definite symmetric matrices $X^{k}=\operatorname{diag}\left\{X_{h}^{k}, X_{v}^{k}\right\}$ and $X^{k l}=$ $\operatorname{diag}\left\{X_{h}^{k l}, X_{v}^{k l}\right\}$ with appropriate dimensions, and positive scalars $\varepsilon_{k}$ and $\varepsilon_{k l}$ such that, for $k, l \in \underline{N}, k \neq l$, the following inequalities hold:

$$
\begin{gathered}
{\left[\begin{array}{ccc}
-\alpha X^{k} & \left(A^{k} X^{k}+B^{k} W^{k}\right)^{T} & \left(E_{1}^{k} X^{k}+E_{3}^{k} W^{k}\right)^{T} \\
* & -X^{k}+\varepsilon_{k} H^{k} H^{k T} & 0 \\
* & * & -\varepsilon_{k} I
\end{array}\right]<0,} \\
{\left[\begin{array}{ccc}
-\beta X^{k l} & \left(A^{l} X^{k l}+B^{l} K^{k} X^{k l}\right)^{T} & \left(E_{1}^{l} X^{k l}+E_{3}^{l} K^{k} X^{k l}\right)^{T} \\
* & -X^{k l}+\varepsilon_{k l} H^{l} H^{l T} & 0 \\
* & * & -\varepsilon_{k l} I
\end{array}\right]<0,}
\end{gathered}
$$

then under the following switching controller:

$$
u(i, j)=K^{\sigma^{\prime}(i, j)} x(i, j), \quad K^{k}=W^{k}\left(X^{k}\right)^{-1},
$$

and the following average dwell time scheme:

$$
\tau_{a}>\tau_{a}^{*}=\frac{\Delta(\ln \beta-\ln \alpha)+\ln \left(\mu_{1} \mu_{2}\right)}{-\ln \alpha},
$$

the resulting closed-loop system (24) is exponentially stable, where $\mu_{1} \mu_{2} \geq 1$ satisfies

$$
X_{l}^{-1} \leq \mu_{1} X_{k l}^{-1}, \quad X_{k l}^{-1} \leq \mu_{2} X_{k}^{-1} .
$$

Furthermore, it should also be noted that if the criterion in Theorem 12 is satisfied when $\Delta=0$, which means that the controller and the subsystem are synchronous, in other words, the results presented in Theorem 12 can be reduced to the synchronous case, then we can obtain the following corollary.

Corollary 17. Consider system (1) under synchronous switching, for a given positive scalar $\alpha<1$, if there exist positive definite symmetric matrices $X^{k}=\operatorname{diag}\left\{X_{h}^{k}, X_{v}^{k}\right\}, Y^{k}=\operatorname{diag}\left\{Y_{h}^{k}\right.$, $\left.Y_{v}^{k}\right\}$, and $W^{k}$, with appropriate dimensions, and a positive scalar $\varepsilon_{k}$, such that, for $k \in \underline{N}$, the following inequality holds:

$$
\left[\begin{array}{ccccc}
-\alpha X^{k} & 0 & \left(A^{k} X^{k}+B^{k} W^{k}\right)^{T} & X^{k} & \left(E_{1}^{k} X^{k}+E_{3}^{k} W^{k}\right)^{T} \\
* & -\Lambda_{1} Y^{k} & \left(A_{d}^{k} Y^{k}\right)^{T} & 0 & \left(E_{2}^{k} Y^{k}\right)^{T} \\
* & * & -X^{k}+\varepsilon_{k} H^{k} H^{k T} & 0 & 0 \\
* & * & * & -Y^{k} & 0 \\
* & * & * & * & -\varepsilon_{k} I
\end{array}\right]<0
$$

then under the following controller:

$$
u(i, j)=K^{k} x(i, j), \quad K^{k}=W^{k}\left(X^{k}\right)^{-1},
$$

and the following average dwell time scheme:

$$
\tau_{a}>\tau_{a}^{*}=\frac{\ln \mu_{1}}{-\ln \alpha}
$$

the resulting closed-loop system is exponentially stable, where $\mu_{1} \geq 1$ satisfies

$$
X_{l}^{-1} \leq \mu_{1} X_{k}^{-1}, \quad Y_{l}^{-1} \leq \mu_{1} Y_{k}^{-1}
$$

Remark 18. In [30], by using the average dwell time approach, a criterion of exponential stability for a class of 2D discrete delay-free switched systems is developed. However, the focus of our work is on stability analysis and controller design under asynchronous switching, which is different from [30], and this is also the major contribution of our work. In fact, if we let $\Delta=0$ and do not consider the uncertainties and state delays, then the closed-loop system (24) is the same as (36) in [30]. In this case, Corollary 17 can be reduced to Theorem 2 in $[30]$.

\section{Numerical Example}

In this section, we present an example to illustrate the effectiveness of the proposed approach.

Consider system (1) with the following parameters:

$$
\begin{aligned}
& A^{1}=\left[\begin{array}{ll}
1 & 1.5 \\
1 & 0.5
\end{array}\right], \quad A_{d}^{1}=\left[\begin{array}{cc}
-0.15 & 0 \\
-0.1 & -0.12
\end{array}\right] \\
& B^{1}=\left[\begin{array}{cc}
-4.5 & 0 \\
1 & -3
\end{array}\right], \quad H^{1}=\left[\begin{array}{cc}
0.2 & 0.15 \\
0.1 & 0.2
\end{array}\right] \text {, }
\end{aligned}
$$




$$
\begin{gathered}
E_{1}^{1}=\left[\begin{array}{cc}
-0.2 & 0 \\
-0.2 & -0.2
\end{array}\right], \quad E_{2}^{1}=\left[\begin{array}{cc}
0.1 & 0 \\
0.1 & 0.2
\end{array}\right], \\
F^{1}=\operatorname{diag}\{\sin (0.5 \pi(i+j)), \sin (0.5 \pi(i+j))\}, \\
E_{3}^{1}=\left[\begin{array}{cc}
0.15 & 0 \\
0.13 & 0.12
\end{array}\right], \quad A^{2}=\left[\begin{array}{ll}
1 & 2 \\
1 & 1
\end{array}\right], \\
A_{d}^{2}=\left[\begin{array}{cc}
-0.1 & 0.2 \\
0 & -0.2
\end{array}\right], \quad B^{2}=\left[\begin{array}{cc}
-5 & 1 \\
-1 & -3
\end{array}\right], \\
H^{2}=\left[\begin{array}{cc}
0.2 & 0.25 \\
0.2 & 0.3
\end{array}\right], \quad E_{1}^{2}=\left[\begin{array}{cc}
0.1 & 0.2 \\
0.2 & 0.1
\end{array}\right] \\
E_{2}^{2}=\left[\begin{array}{cc}
0.2 & 0.1 \\
0.2 & 0.1
\end{array}\right], \quad E_{3}^{2}=\left[\begin{array}{cc}
0.12 & 0.15 \\
0.12 & 0.1
\end{array}\right], \\
F^{2}=\operatorname{diag}\{\cos (0.5 \pi(i+j)), \cos (0.5 \pi(i+j))\}, \\
d_{h}=2, \quad d_{v}=3 .
\end{gathered}
$$

The boundary conditions are given as follows:

$$
\begin{aligned}
x^{h}(0, j) & = \begin{cases}5, & 0 \leq j \leq 20 \\
0, & j>20,\end{cases} \\
x^{v}(i, 0) & = \begin{cases}3, & 0 \leq i \leq 20 \\
0, & i>20\end{cases}
\end{aligned}
$$

where the state dimensions are $n_{1}=1$ and $n_{2}=1$.

Take $\alpha=0.6$ and $\beta=1.2$, then solving (19) in Theorem 12 gives rise to

$$
\begin{array}{rlr}
X^{1}=\left[\begin{array}{cc}
36.3903 & 1.0247 \\
1.0247 & 38.3909
\end{array}\right], & X^{2}=\left[\begin{array}{ll}
40.0833 & -6.6640 \\
-6.6640 & 34.4516
\end{array}\right], \\
Y^{1}=\left[\begin{array}{cc}
96.6213 & -4.5896 \\
-4.5896 & 96.9472
\end{array}\right], & Y^{2}=\left[\begin{array}{ll}
99.6213 & -8.8472 \\
-8.8472 & 79.5315
\end{array}\right], \\
W^{1}=\left[\begin{array}{cc}
8.5866 & 13.0604 \\
15.5568 & 11.4091
\end{array}\right], & W^{2}=\left[\begin{array}{cc}
6.9844 & 13.2930 \\
8.3790 & 4.4234
\end{array}\right], \\
\varepsilon_{1}=74.2107, & \varepsilon_{2}=73.4149 .
\end{array}
$$

By (21), $K^{1}$ and $K^{2}$ can be obtained as follows:

$$
K^{1}=\left[\begin{array}{ll}
0.2265 & 0.3341 \\
0.4194 & 0.2860
\end{array}\right], \quad K^{2}=\left[\begin{array}{ll}
0.2463 & 0.4335 \\
0.2380 & 0.1744
\end{array}\right] .
$$

Substituting $K^{1}$ and $K^{2}$ into (20), and solving it, we get the following solution:

$$
\begin{array}{rlrl}
X^{12} & =\left[\begin{array}{cc}
0.5400 & -0.0934 \\
-0.0934 & 0.4811
\end{array}\right], & X^{21} & =\left[\begin{array}{cc}
0.5805 & -0.0926 \\
-0.0926 & 0.5677
\end{array}\right], \\
Y^{12} & =\left[\begin{array}{cc}
0.9398 & -0.0418 \\
-0.0418 & 0.8451
\end{array}\right], & Y^{21} & =\left[\begin{array}{cc}
0.9436 & -0.0340 \\
-0.0340 & 0.8867
\end{array}\right], \\
\varepsilon_{12}=0.9011, & \varepsilon_{21}=0.7725
\end{array}
$$

Then, from (23), we can get that $\mu_{1}=0.0171$ and $\mu_{2}=$ 917.7224. Taking $\Delta=2$, it is easy to obtain from (22) that $\tau_{a}^{*}=7.9$. Choosing $\tau_{a}=8$, the trajectories of the states $x^{h}(i, j)$ and $x^{v}(i, j)$ are shown in Figures 1 and 2, respectively. The

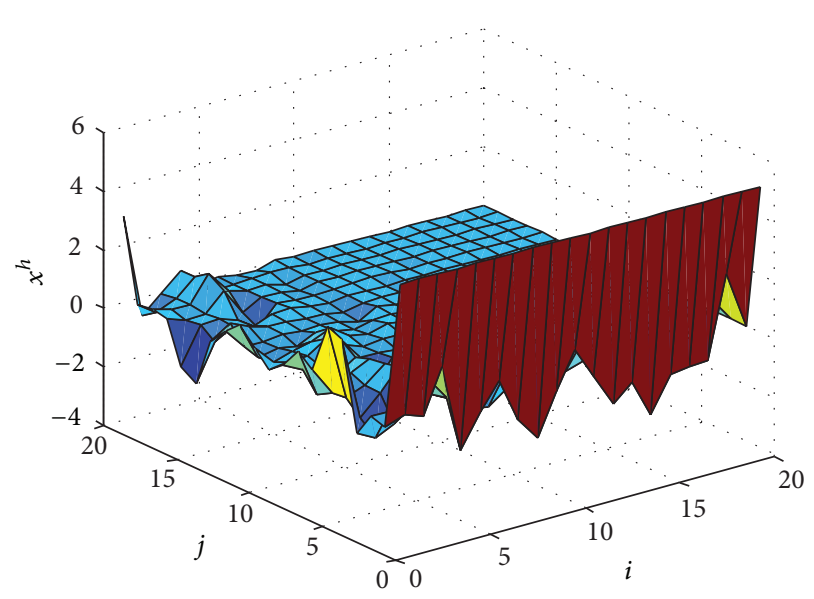

FIGURE 1: The trajectory of the state $x^{h}(i, j)$.

system switching signal $\sigma(i, j)$ and the controller switching signal $\sigma^{\prime}(i, j)$ are shown in Figure 3 . One can see that the states of the closed-loop system converge to zero under the asynchronous switching. This demonstrates the effectiveness of the proposed approach.

\section{Conclusions}

This paper has investigated the problem of stabilization for a class of 2D discrete switched systems with constant state delays under asynchronous switching. A state feedback controller is proposed to stabilize such system, and the dwell time approach is utilized for the stability analysis and controller design. A sufficient condition for the existence of such controller is formulated in terms of a set of LMIs. An example is also given to illustrate the applicability of the proposed approach. Our future work will focus on extending the proposed design method to other problems such as robust $H_{\infty}$ control for $2 \mathrm{D}$ discrete switched systems with time-varying delays and fractional uncertainties under asynchronous switching.

\section{Appendix}

Proof of Lemma 9. Consider the following Lyapunov-Krasovskii functional candidate:

$$
V(x(i, j))=V^{h}\left(x^{h}(i, j)\right)+V^{v}\left(x^{v}(i, j)\right),
$$

where

$$
\begin{aligned}
& V^{h}\left(x^{h}(i, j)\right) \\
& =x^{h}(i, j)^{T} P_{h} x^{h}(i, j) \\
& \quad+\sum_{r=i-d_{h}}^{i-1} x^{h}(r, j)^{T} Q_{h} x^{h}(r, j) \alpha^{i-r-1}, \\
& V^{v}\left(x^{v}(i, j)\right) \\
& =x^{v}(i, j)^{T} P_{v} x^{v}(i, j) \\
& \quad+\sum_{t=j-d_{v}}^{j-1} x^{v}(i, t)^{T} Q_{v} x^{v}(i, t) \alpha^{j-t-1} .
\end{aligned}
$$




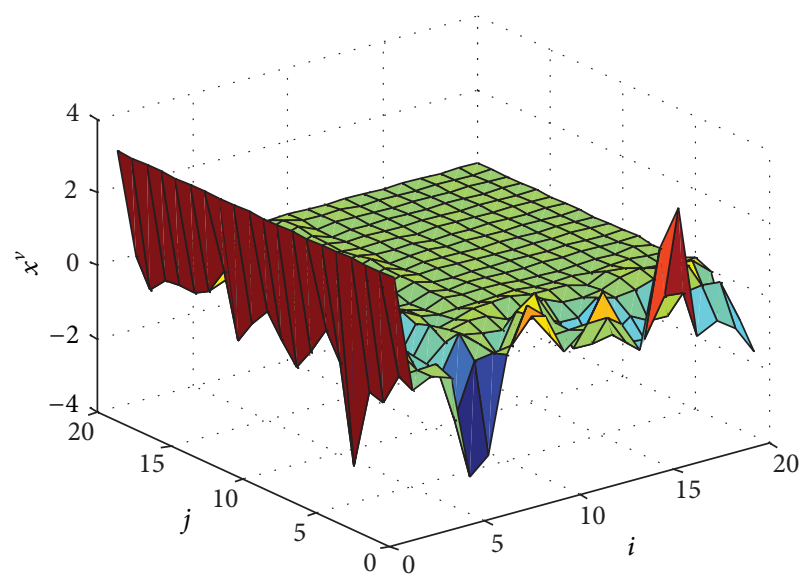

Figure 2: The trajectory of the state $x^{v}(i, j)$.

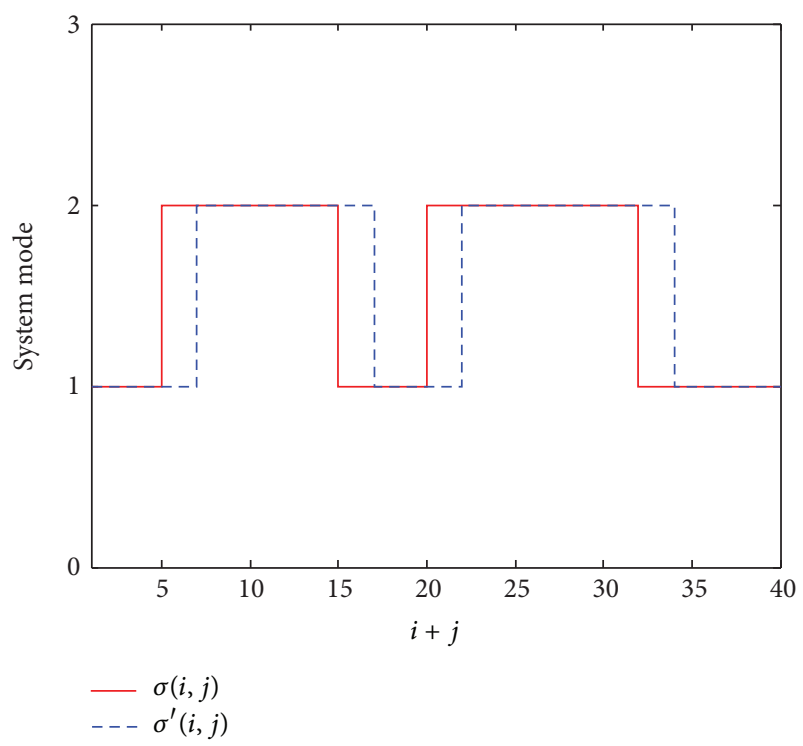

Figure 3: Switching signal.

Along the trajectory of system (8), we have

$$
\begin{aligned}
& V^{h}\left(x^{h}(i+1, j)\right)-\alpha V^{h}\left(x^{h}(i, j)\right) \\
&= x^{h}(i+1, j)^{T} P_{h} x^{h}(i+1, j)-\alpha x^{h}(i, j)^{T} P_{h} x^{h}(i, j) \\
&+x^{h}(i, j)^{T} Q_{h} x^{h}(i, j) \\
&-\alpha^{d_{h}} x^{h}\left(i-d_{h}, j\right)^{T} Q_{h} x^{h}\left(i-d_{h}, j\right), \\
& V^{v}\left(x^{v}(i, j+1)\right)-\alpha V^{v}\left(x^{v}(i, j)\right) \\
&=x^{v}(i, j+1)^{T} P_{v} x^{v}(i, j+1)-\alpha x^{v}(i, j)^{T} P_{v} x^{v}(i, j) \\
& \quad+x^{v}(i, j)^{T} Q_{v} x^{v}(i, j) \\
& \quad-\alpha^{d_{v}} x^{v}\left(i, j-d_{v}\right)^{T} Q_{v} x^{v}\left(i, j-d_{v}\right) .
\end{aligned}
$$

It follows that

$$
\begin{aligned}
V^{h}\left(x^{h}(i+1, j)\right)-\alpha V^{h}\left(x^{h}(i, j)\right) & +V^{v}\left(x^{v}(i, j+1)\right)-\alpha V^{v}\left(x^{v}(i, j)\right) \\
= & {\left[\begin{array}{c}
{\left[\begin{array}{l}
x^{h}(i, j) \\
x^{v}(i, j)
\end{array}\right]} \\
{\left[\begin{array}{l}
x^{h}\left(i-d_{h}, j\right) \\
x^{h}\left(i, j-d_{v}\right)
\end{array}\right]}
\end{array}\right]\left[\begin{array}{ll}
\Phi_{11} & \Phi_{12} \\
\Phi_{12}^{T} & \Phi_{22}
\end{array}\right]\left[\begin{array}{c}
{\left[\begin{array}{l}
x^{h}(i, j) \\
x^{v}(i, j)
\end{array}\right]} \\
{\left[\begin{array}{l}
x^{h}\left(i-d_{h}, j\right) \\
x^{h}\left(i, j-d_{v}\right)
\end{array}\right]}
\end{array}\right], }
\end{aligned}
$$

where

$$
\begin{gathered}
\Phi_{11}=Q-\alpha P+\widehat{A}^{T} P \widehat{A}, \quad \Phi_{12}=\widehat{A}^{T} P \widehat{A}_{d} \\
\Phi_{22}=\widehat{A}_{d}^{T} P \widehat{A}_{d}-\Lambda_{1} Q, \quad \Lambda_{1}=\operatorname{diag}\left\{\alpha^{d_{h}} I_{h}, \alpha^{d_{v}} I_{v}\right\} .
\end{gathered}
$$

Applying Lemma 7, it can be obtained from (14) that

$$
\left[\begin{array}{ll}
\Phi_{11} & \Phi_{12} \\
\Phi_{12}^{T} & \Phi_{22}
\end{array}\right]<0 .
$$

For simplicity, we denote

$$
\begin{gathered}
V^{h}(i, j)=V^{h}\left(x^{h}(i, j)\right), \quad V^{v}(i, j)=V^{v}\left(x^{v}(i, j)\right), \\
V(i, j)=V(x(i, j)), \quad V^{h}(i+1, j)=V^{h}(x(i+1, j)), \\
V^{v}(i, j+1)=V^{v}(x(i, j+1)) .
\end{gathered}
$$

Thus, it is easy to get that

$$
V^{h}(i+1, j)+V^{v}(i, j+1)<\alpha\left(V^{h}(i, j)+V^{v}(i, j)\right) .
$$

Notice that for any nonnegative integer $D>z=\max \left(z_{1}, z_{2}\right)$, it holds that $V^{h}(0, D)=V^{v}(D, 0)=0$; then summing up both sides of (A.9) from $D-1$ to 0 with respect to $j$ and 0 to $D-1$ with respect to $i$, for any nonnegative integer $D>D^{\prime} \geq z=$ $\max \left(z_{1}, z_{2}\right)$, one gets

$$
\begin{aligned}
\sum_{i+j=D} V(i, j) & \\
= & V^{h}(0, D)+V^{h}(1, D-1)+V^{h}(2, D-2) \\
& +\cdots+V^{h}(D-1,1)+V^{h}(D, 0) \\
& +V^{v}(0, D)+V^{v}(1, D-1)+V^{v}(2, D-2) \\
& +\cdots+V^{v}(D-1,1)+V^{v}(D, 0) \\
< & \alpha\left(V^{h}(0, D-1)+V^{v}(0, D-1)\right. \\
& +V^{h}(1, D-2)+V^{v}(1, D-2) \\
& \left.+\cdots+V^{h}(D-1,0)+V^{v}(D-1,0)\right) \\
= & \alpha \sum_{i+j=D-1} V(i, j)<\cdots<\alpha^{D-D^{\prime}} \sum_{i+j=D^{\prime}} V(i, j) .
\end{aligned}
$$

The proof is completed. 
Proof of Theorem 12. When $D \in\left[m_{\kappa}+\Delta m_{\kappa}, m_{\kappa+1}\right)$, the closed-loop system (17) can be written as

$$
\begin{aligned}
& {\left[\begin{array}{l}
x^{h}(i+1, j) \\
x^{v}(i, j+1)
\end{array}\right]} \\
& \quad=\left(\widehat{A}^{k}+\widehat{B}^{k} K^{k}\right)\left[\begin{array}{c}
x^{h}(i, j) \\
x^{v}(i, j)
\end{array}\right]+\widehat{A}_{d}^{k}\left[\begin{array}{c}
x^{h}\left(i-d_{h}, j\right) \\
x^{v}\left(i, j-d_{v}\right)
\end{array}\right] .
\end{aligned}
$$

For the system, we consider the following Lyapunov function candidate:

$$
V_{k}(x(i, j))=V_{k}^{h}\left(x^{h}(i, j)\right)+V_{k}^{v}\left(x^{v}(i, j)\right)
$$

where

$$
\begin{aligned}
& V_{k}^{h}\left(x^{h}(i, j)\right) \\
& =x^{h}(i, j)^{T} P_{h}^{k} x^{h}(i, j)+\sum_{r=i-d_{h}}^{i-1} x^{h}(r, j)^{T} Q_{h}^{k} x^{h}(r, j) \alpha^{i-r-1}, \\
& V_{k}^{v}\left(x^{v}(i, j)\right) \\
& =x^{v}(i, j)^{T} P_{v}^{k} x^{v}(i, j)+\sum_{t=j-d_{v}}^{j-1} x^{v}(i, t)^{T} Q_{v}^{k} x^{v}(i, t) \alpha^{j-t-1} .
\end{aligned}
$$

By Lemma 9, one gets that if there exist positive definite symmetric matrices $P^{k}=\operatorname{diag}\left\{P_{h}^{k}, P_{v}^{k}\right\}$ and $Q^{k}=\operatorname{diag}\left\{Q_{h}^{k}, Q_{v}^{k}\right\}$ with appropriate dimensions, such that

$$
\left[\begin{array}{ccc}
Q^{k}-\alpha P^{k} & 0 & \left(\widehat{A}^{k}+\widehat{B}^{k} K^{k}\right)^{T} P^{k} \\
* & -\Lambda_{1} Q^{k} & \widehat{A}_{d}^{k T} P^{k} \\
* & * & -P^{k}
\end{array}\right]<0
$$

holds, then the following inequality holds for any $D \geq m_{\kappa}+$ $\Delta m_{\kappa} \geq z$ :

$$
\sum_{i+j=D} V_{k}(i, j)<\alpha^{D-m_{k}-\Delta m_{k}} \sum_{i+j=m_{\kappa}+\Delta m_{\kappa}} V_{k}(i, j) .
$$

When $D \in\left[m_{\kappa+1}, m_{\kappa+1}+\Delta m_{\kappa+1}\right)$, the closed-loop system (17) can be written as

$$
\begin{aligned}
& {\left[\begin{array}{l}
x^{h}(i+1, j) \\
x^{v}(i, j+1)
\end{array}\right]} \\
& =\left(\widehat{A}^{l}+\widehat{B}^{l} K^{k}\right)\left[\begin{array}{l}
x^{h}(i, j) \\
x^{v}(i, j)
\end{array}\right]+\widehat{A}_{d}^{l}\left[\begin{array}{l}
x^{h}\left(i-d_{h}, j\right) \\
x^{v}\left(i, j-d_{v}\right)
\end{array}\right] .
\end{aligned}
$$

Consider the following Lyapunov function candidate:

$$
V_{k l}(x(i, j))=V_{k l}^{h}\left(x^{h}(i, j)\right)+V_{k l}^{v}\left(x^{v}(i, j)\right),
$$

where

$$
\begin{aligned}
& V_{k l}^{h}(\left.x^{h}(i, j)\right) \\
&= x^{h}(i, j)^{T} P_{h}^{k l} x^{h}(i, j) \\
&+\sum_{r=i-d_{h}}^{i-1} x^{h}(r, j)^{T} Q_{h}^{k l} x^{h}(r, j) \alpha^{i-r-1}, \\
& V_{k l}^{v}\left(x^{v}(i, j)\right) \\
&=x^{v}(i, j)^{T} P_{v}^{k l} x^{v}(i, j) \\
& \quad+\sum_{t=j-d_{v}}^{j-1} x^{v}(i, t)^{T} Q_{v}^{k l} x^{v}(i, t) \alpha^{j-t-1} .
\end{aligned}
$$

Similarly, by Lemma 9, we get that if there exist positive definite symmetric matrices $P^{k l}=\operatorname{diag}\left\{P_{h}^{k l}, P_{v}^{k l}\right\}$ and $Q^{k l}=$ $\operatorname{diag}\left\{Q_{h}^{k l}, Q_{v}^{k l}\right\}$ with appropriate dimensions, such that

$$
\left[\begin{array}{ccc}
Q^{k l}-\beta P^{k l} & 0 & \left(\widehat{A}^{l}+\widehat{B}^{l} K^{k}\right)^{T} P^{k l} \\
* & -\Lambda_{2} Q^{k l} & \widehat{A}_{d}^{l T} P^{k l} \\
* & * & -P^{k l}
\end{array}\right]<0
$$

holds, then the following inequality holds for any $D \geq m_{\kappa+1} \geq$ $z$ :

$$
\sum_{i+j=D} V_{k l}(i, j)<\beta^{D-m_{k+1}} \sum_{i+j=m_{\kappa+1}} V_{k l}(i, j)
$$

Consider the following piecewise Lyapunov functional candidate for system (17):

$$
\left\{\begin{array}{l}
x^{h}(i, j)^{T} P_{h}^{\sigma(i, j)} x^{h}(i, j) \\
\quad+x^{v}(i, j)^{T} P_{v}^{\sigma(i, j)} x^{v}(i, j) \\
\quad+\sum_{r=i-d_{h}}^{i-1} x^{h}(r, j)^{T} Q_{h}^{\sigma(i, j)} x^{h}(r, j) \alpha^{i-r-1} \\
\quad+\sum_{t=j-d_{v}}^{j-1} x^{v}(i, t)^{T} Q_{v}^{\sigma(i, j)} x^{v}(i, t) \alpha^{j-t-1}, \\
D \in\left[m_{0}, m_{1}\right) \cup\left[m_{\pi}+\Delta m_{\pi}, m_{\pi+1}\right), \\
\quad+x^{\nu}(i, j)^{T} P_{v}^{\sigma^{\prime}(i, j) \sigma(i, j)} x^{v}(i, j) \\
\quad+\sum_{r=i-d_{h}}^{i-1} x^{h}(r, j)^{T} Q_{h}^{\sigma^{\prime}(i, j) \sigma(i, j)} x^{h}(r, j) \alpha^{i-r-1} \\
\quad+\sum_{t=j-d_{v}}^{j-1} x^{v}(i, t)^{T} Q_{v}^{\sigma^{\prime}(i, j) \sigma(i, j)} x^{v}(i, t) \alpha^{j-t-1}, \\
D \in\left[m_{\pi}, m_{\pi}+\Delta m_{\pi}\right), \quad \pi=1,2, \ldots, \kappa \ldots
\end{array}\right.
$$


Now, let $v=N_{\sigma(i, j)}(z, D)$ denote the switch number of $\sigma(i, j)$ on the interval $[z, D)$, and let $\left(i_{\kappa-v+1}, j_{\kappa-v+1}\right)$, $\left(i_{\kappa-v+2}, j_{\kappa-v+2}\right), \ldots,\left(i_{\kappa}, j_{\kappa}\right)$ denote the switching points of $\sigma(i, j)$ over the interval $[z, D)$; then, the switching points of $\sigma^{\prime}(i, j)$ can be denoted as follows:

$$
\begin{gathered}
\left(i_{\kappa-v+1}+\Delta i_{\kappa-v+1}, j_{\kappa-v+1}+\Delta j_{\kappa-v+1}\right) \\
\left(i_{\kappa-v+2}+\Delta i_{\kappa-v+2}, j_{\kappa-v+2}+\Delta j_{\kappa-v+2}\right), \ldots,\left(i_{\kappa}+\Delta i_{\kappa}, j_{\kappa}+\Delta j_{\kappa}\right) .
\end{gathered}
$$

Denoting $m_{g}=i_{g}+j_{g}, g=\kappa-v+1, \ldots, \kappa$, then we can get from (23) and (A.21) that

$$
\begin{gathered}
\sum_{i+j=m_{g}+\Delta m_{g}} V(i, j) \leq \mu_{1} \sum_{i+j=\left(m_{g}+\Delta m_{g}\right)^{-}} V(i, j), \\
\sum_{i+j=m_{g}} V(i, j) \leq \mu_{2} \sum_{i+j=\left(m_{g}\right)^{-}} V(i, j),
\end{gathered}
$$

where $\left(m_{g}\right)^{-}$and $\left(m_{g}+\Delta m_{g}\right)^{-}$satisfy the following conditions:

$$
\begin{gathered}
0<m_{g}-\left(m_{g}\right)^{-}<\lambda, \\
0<m_{g}+\Delta m_{g}-\left(m_{g}+\Delta m_{g}\right)^{-}<\lambda,
\end{gathered}
$$

where $\lambda$ is a sufficient small positive constant.

When $m_{\kappa-v+1}>z \geq m_{\kappa-v}+\Delta m_{\kappa-v}$, we have, for $D>$ $m_{\kappa}+\Delta m_{\kappa}$,

$$
\begin{aligned}
& \sum_{i+j=D} V(i, j)<\alpha^{D-m_{\kappa}-\Delta m_{\kappa}} \sum_{i+j=m_{\kappa}+\Delta m_{\kappa}} V(i, j) \leq \mu_{1} \alpha^{D-m_{\kappa}-\Delta m_{\kappa}} \sum_{i+j=\left(m_{\kappa}+\Delta m_{\kappa}\right)^{-}} V(i, j) \\
& <\mu_{1} \alpha^{D-m_{\kappa}-\Delta m_{\kappa}} \beta^{\Delta m_{\kappa}} \sum_{i+j=m_{\kappa}} V(i, j) \leq \mu_{1} \mu_{2} \alpha^{D-m_{\kappa}-\Delta m_{\kappa}} \beta^{\Delta m_{\kappa}} \sum_{i+j=\left(m_{\kappa}\right)^{-}} V(i, j)<\cdots \\
& <\left(\mu_{1} \mu_{2}\right)^{v} \alpha^{D-m_{\kappa}-\Delta m_{\kappa}+m_{\kappa}-m_{\kappa-1}-\Delta m_{\kappa-1}+\cdots+m_{\kappa-v+2}-m_{\kappa-v+1}-\Delta m_{\kappa-v+1}} \beta^{\Delta m_{\kappa}+\Delta m_{\kappa-1}+\cdots+\Delta m_{\kappa-v+1}} \sum_{i+j=\left(m_{k-v+1}\right)^{-}} V(i, j) \\
& <\left(\mu_{1} \mu_{2}\right)^{v} \alpha^{D-m_{\kappa}-\Delta m_{\kappa}+m_{\kappa}-m_{\kappa-1}-\Delta m_{\kappa-1}+\cdots+m_{\kappa-v+2}-m_{\kappa-v+1}-\Delta m_{\kappa-v+1}+m_{\kappa-v+1}-z} \beta^{\Delta m_{\kappa}+\Delta m_{\kappa-1}+\cdots+\Delta m_{\kappa-v+1}} \sum_{i+j=z} V(i, j) \\
& \leq\left(\mu_{1} \mu_{2}\right)^{v} \alpha^{D-z-v \Delta} \beta^{v \Delta} \sum_{i+j=z} V(i, j)=\alpha^{D-z} e^{v \ln \left(\mu_{1} \mu_{2}\right)+v \Delta(\ln \beta-\ln \alpha)} \sum_{i+j=z} V(i, j) .
\end{aligned}
$$

When $m_{\kappa-v}<z<m_{\kappa-v}+\Delta m_{\kappa-v}$, we can also get that

$$
\begin{aligned}
& \sum_{i+j=D} V(i, j)<\alpha^{D-m_{\kappa}-\Delta m_{\kappa}} \sum_{i+j=m_{\kappa}+\Delta m_{\kappa}} V(i, j)<\mu_{1} \alpha^{D-m_{\kappa}-\Delta m_{\kappa}} \sum_{i+j=\left(m_{\kappa}+\Delta m_{\kappa}\right)^{-}} V(i, j) \\
& <\mu_{1} \alpha^{D-m_{\kappa}-\Delta m_{\kappa}} \beta^{\Delta m_{\kappa}} \sum_{i+j=m_{\kappa}} V(i, j)<\mu_{1} \mu_{2} \alpha^{D-m_{\kappa}-\Delta m_{\kappa}} \beta^{\Delta m_{\kappa}} \sum_{i+j=\left(m_{\kappa}\right)^{-}} V(i, j)<\cdots \\
& <\left(\mu_{1} \mu_{2}\right)^{v} \alpha^{D-m_{\kappa}-\Delta m_{\kappa}+m_{\kappa}-m_{\kappa-1}-\Delta m_{\kappa-1}+\cdots+m_{\kappa-v+2}-m_{\kappa-v+1}-\Delta m_{\kappa-v+1}} \beta^{\Delta m_{\kappa}+\Delta m_{\kappa-1}+\ldots+\Delta m_{\kappa-v+1}} \sum_{i+j=\left(m_{k-v+1}\right)^{-}} V(i, j) \\
& <\left(\mu_{1} \mu_{2}\right)^{v} \alpha^{D-m_{\kappa}-\Delta m_{\kappa}+m_{\kappa}-m_{\kappa-1}-\Delta m_{\kappa-1}+\cdots+m_{\kappa-v+2}-m_{\kappa-v+1}-\Delta m_{\kappa-v+1}+m_{\kappa-v+1}-m_{\kappa-v}-\Delta m_{\kappa-v}} \beta^{\Delta m_{\kappa}+\Delta m_{\kappa-1}+\cdots+\Delta m_{\kappa-v+1}+\Delta m_{\kappa-v}+m_{\kappa-v}-z} \sum_{i+j=z} V(i, j) \\
& \leq \mu_{1}\left(\mu_{1} \mu_{2}\right)^{v} \alpha^{D-z-v \Delta} \beta^{v \Delta} \sum_{i+j=z} V(i, j)=\mu_{1} \alpha^{D-z} e^{v \ln \left(\mu_{1} \mu_{2}\right)+v \Delta(\ln \beta-\ln \alpha)} \sum_{i+j=z} V(i, j) .
\end{aligned}
$$

From (A.25) and (A.26), we can obtain

$$
\begin{aligned}
& \sum_{i+j=D} V(i, j) \\
& \quad<\max \left\{\mu_{1}, 1\right\} \alpha^{D-z} e^{v \ln \left(\mu_{1} \mu_{2}\right)+\Delta v(\ln \beta-\ln \alpha)} \sum_{i+j=z} V(i, j) .
\end{aligned}
$$

According to Definition 6, one has

$$
v=N_{\sigma(i, j)}(z, D) \leq N_{0}+\frac{D-z}{\tau_{a}} .
$$

From condition (22), the following inequality can be obtained:

$$
-\ln \alpha>\frac{\Delta(\ln \beta-\ln \alpha)+\ln \left(\mu_{1} \mu_{2}\right)}{\tau_{a}} .
$$


Thus, it is easy to get the following inequality:

$$
\begin{aligned}
& \sum_{i+j=D} V(i, j) \\
& <\max \left\{\mu_{1}, 1\right\}\left(\mu_{1} \mu_{2}\right)^{N_{0}} \\
& \quad \times\left(\frac{\beta}{\alpha}\right)^{N_{0} \Delta} e^{\left(\left(\ln \left(\mu_{1} \mu_{2}\right)+\Delta(\ln \beta-\ln \alpha)\right) / \tau_{a}+\ln \alpha\right)(D-z)} \sum_{i+j=z} V(i, j) .
\end{aligned}
$$

It follows that

$$
\begin{aligned}
& \sum_{i+j=D}\|x(i, j)\|^{2} \\
& <\frac{\zeta_{1}}{\zeta_{2}} \max \left\{\mu_{1}, 1\right\}\left(\mu_{1} \mu_{2}\right)^{N_{0}} \\
& \quad \times\left(\frac{\beta}{\alpha}\right)^{N_{0} \Delta} e^{\left(\left(\ln \left(\mu_{1} \mu_{2}\right)+\Delta(\ln \beta-\ln \alpha)\right) / \tau_{a}+\ln \alpha\right)(D-z)} \sum_{i+j=z}\|x(i, j)\|^{2},
\end{aligned}
$$

where

$$
\begin{gathered}
\zeta_{1}=\max _{k, l \in \underline{N}, k \neq l}\left\{\lambda_{\max }\left(P^{k}\right)+\max \left(d_{h}, d_{v}\right) \lambda_{\max }\left(Q^{k}\right),\right. \\
\left.\lambda_{\max }\left(P^{k l}\right)+\max \left(d_{h}, d_{v}\right) \lambda_{\max }\left(Q^{k l}\right)\right\}, \\
\zeta_{2}=\min _{k, l \in \underline{N}, k \neq l}\left\{\lambda_{\min }\left(P^{k}\right), \lambda_{\min }\left(P^{k l}\right)\right\}, \\
\sum_{i+j=z}\|x(i, j)\|_{C}^{2} \\
\triangleq \sup _{\substack{-d_{h} \leq \theta_{h} \leq 0,-d_{v} \leq \theta_{v} \leq 0}} \sum_{i+j=z}\left\{\left\|x^{h}\left(i-\theta_{h}, j\right)\right\|^{2}+\left\|x^{v}\left(i, j-\theta_{v}\right)\right\|^{2}\right\},
\end{gathered}
$$

Thus, it can be obtained from (22) that the closed-loop system (17) is exponentially stable.

Denote $X^{k}=\left(P^{k}\right)^{-1}$ and $Y^{k}=\left(Q^{k}\right)^{-1}$, then it is easy to get $\left(X^{k}\right)^{T}=X^{k}$ and $\left(Y^{k}\right)^{T}=Y^{k}$. Using $\operatorname{diag}\left\{X^{k}, Y^{k}, X^{k}\right\}$ to preand postmultiply the left of (A.14), respectively, and applying Lemma 7, it follows that (A.33) and (A.14) are equivalent:

$$
\left[\begin{array}{cccc}
-\alpha X^{k} & 0 & \left(\widehat{A}^{k} X^{k}+\widehat{B}^{k} W^{k}\right)^{T} & X^{k} \\
* & -\Lambda_{1} Y^{k} & \left(\widehat{A}_{d}^{k} Y^{k}\right)^{T} & 0 \\
* & * & -X^{k} & 0 \\
* & * & * & -Y^{k}
\end{array}\right]<0
$$

where $W^{k}=K^{k} X^{k}$.

By substituting (2) into (A.33), we can get the following inequality

$$
T=T_{0}+T_{1}<0
$$

where

$$
\begin{aligned}
& T_{0}=\left[\begin{array}{cccc}
-\alpha X^{k} & 0 & \left(A^{k} X^{k}+B^{k} W^{k}\right)^{T} & X^{k} \\
* & -\Lambda_{1} Y^{k} & \left(A_{d}^{k} Y^{k}\right)^{T} & 0 \\
* & * & -X^{k} & 0 \\
* & * & * & -Y^{k}
\end{array}\right], \\
& T_{1}=\left[\begin{array}{c}
\left(E_{1}^{k} X^{k}+E_{3}^{k} W^{k}\right)^{T} \\
\left(E_{2}^{k} Y^{k}\right)^{T} \\
0 \\
0
\end{array}\right] F^{k T}\left[\begin{array}{c}
0 \\
0 \\
H^{k} \\
0
\end{array}\right]^{T} \\
& +\left[\begin{array}{c}
0 \\
0 \\
H^{k} \\
0
\end{array}\right] F^{k}\left[\begin{array}{c}
\left(E_{1}^{k} X^{k}+E_{3}^{k} W^{k}\right)^{T} \\
\left(E_{2}^{k} Y^{k}\right)^{T} \\
0 \\
0
\end{array}\right]^{T}
\end{aligned}
$$

By Lemma 8, we get

$$
\begin{gathered}
T_{0}+\varepsilon_{k}^{-1}\left[\begin{array}{c}
\left(E_{1}^{k} X^{k}+E_{3}^{k} W^{k}\right)^{T} \\
\left(E_{2}^{k} Y^{k}\right)^{T} \\
0 \\
0
\end{array}\right]\left[\begin{array}{c}
\left(E_{1}^{k} X^{k}+E_{3}^{k} W^{k}\right)^{T} \\
\left(E_{2}^{k} Y^{k}\right)^{T} \\
0 \\
0
\end{array}\right]\left[\begin{array}{c}
0 \\
0 \\
0 \\
H^{k} \\
0
\end{array}\right]\left[\begin{array}{c}
0 \\
0 \\
H^{k} \\
0
\end{array}\right]^{T}<0 .
\end{gathered}
$$

Applying Lemma 7 again, we obtain that (A.33) holds if (19) is satisfied.

Similarly, substitute (2) into (A.19) and denote $X^{k l}=$ $\left(P^{k l}\right)^{-1}$ and $Y^{k l}=\left(Q^{k l}\right)^{-1}$, then using $\operatorname{diag}\left\{X^{k l}, Y^{k l}, X^{k l}\right\}$ to pre- and postmultiply the left of (A.19), respectively, and applying Lemmas 7 and 8, it is easy to get that (A.19) holds if $(20)$ is satisfied.

The proof is completed.

\section{Acknowledgment}

This work was supported by the National Natural Science Foundation of China under Grant no. 61273120.

\section{References}

[1] C. Du and L. Xie, Control and Filtering of Two-Dimensional Systems, vol. 278 of Lecture Notes in Control and Information Sciences, Springer, Berlin, Germany, 2002.

[2] T. Kaczorek, Two-Dimensional Linear Systems, vol. 68 of Lecture Notes in Control and Information Sciences, Springer, Berlin, Germany, 1985.

[3] W. S. Lu, Two-Dimensional Digital Filters, Marcel Dekker, New York, NY, USA, 1992. 
[4] H. Mahgoub and R. Carroll, "A Lyapunov function of twodimensional linear systems," IEEE Transactions on Automatic Control, vol. 31, no. 2, pp. 170-172, 1986.

[5] A. A. Kara and Z. Ünver, "Comments on 'A Lyapunov function of two-dimensional linear systems," IEEE Transaction on Automatic Control, vol. 36, no. 1, pp. 125-126, 1991.

[6] T. Kaczorek, "New stability tests of positive standard and fractional linear systems," Circuits and Systems, vol. 2, no. 4, pp. 261268, 2011.

[7] S. Ye and W. Wang, "Stability analysis and stabilisation for a class of 2-D nonlinear discrete systems," International Journal of Systems Science, vol. 42, no. 5, pp. 839-851, 2011.

[8] W. S. Lu and E. B. Lee, "Stability analysis for two-dimensional systems via a Lyapunov approach," IEEE Transactions on Circuits and Systems, vol. 32, no. 1, pp. 61-68, 1985.

[9] J. Hu, Z. Wang, H. Gao, and L. K. Stergioulas, "Robust sliding mode control for discrete stochastic systems with mixed time delays, randomly occurring uncertainties, and randomly occurring nonlinearities," IEEE Transactions on Industrial Electronics, vol. 59, no. 7, pp. 3008-3015, 2012.

[10] J. Hu, Z. Wang, and H. Gao, "A delay fractioning approach to robust sliding mode control for discrete-time stochastic systems with randomly occurring non-linearities," IMA Journal of Mathematical Control and Information, vol. 28, no. 3, pp. 345363, 2011.

[11] J. Hu, Z. Wang, Y. Niu, and L. K. Stergioulas, " $H_{\infty}$ sliding mode observer design for a class of nonlinear discrete time-delay systems: a delay-fractioning approach," International Journal of Robust and Nonlinear Control, vol. 22, no. 16, pp. 1806-1826, 2012.

[12] J. Xu and L. Yu, " $H_{\infty}$ control of 2-D discrete state delay systems," International Journal of Control, Automation and Systems, vol. 4, no. 4, pp. 516-523, 2006.

[13] S.-F. Chen, "Delay-dependent stability for 2D systems with time-varying delay subject to state saturation in the Roesser model," Applied Mathematics and Computation, vol. 216, no. 9, pp. 2613-2622, 2010.

[14] Z.-Y. Feng, L. Xu, M. Wu, and Y. He, "Delay-dependent robust stability and stabilisation of uncertain two-dimensional discrete systems with time-varying delays," IET Control Theory \& Applications, vol. 4, no. 10, pp. 1959-1971, 2010.

[15] S. F. Chen, "Stability analysis for 2-D systems with interval timevarying delays and saturation nonlinearities," Signal Processing, vol. 90, no. 7, pp. 2265-2275, 2010.

[16] S. Ye, Y. Zou, W. Wang, and J. Yao, "Delay-dependent stability analysis for two- dimensional discrete systems with shift delays by the general models," in Proceedings of the 10th International Conference on Control, Automation, Robotics and Vision (ICARCV '08), pp. 973-978, December 2008.

[17] M. S. Branicky, "Multiple Lyapunov functions and other analysis tools for switched and hybrid systems," IEEE Transactions on Automatic Control, vol. 43, no. 4, pp. 475-482, 1998.

[18] J. P. Hespanha and A. S. Morse, "Stability of switched systems with average dwell-time," in Proceedings of the 38th IEEE Conference on Decision and Control (CDC '99), pp. 2655-2660, December 1999.

[19] G. Xie and L. Wang, "Stabilization of switched linear systems with time-delay in detection of switching signal," Journal of Mathematical Analysis and Applications, vol. 305, no. 1, pp. 277290, 2005.
[20] Y. Wang, Z. Yao, Z. Zuo, and H. Zhao, "Delay-dependent robust $H_{\infty}$ control for a class of switched systems with time delay," in Proceedings of IEEE International Symposium on Intelligent Control (ISIC '08), pp. 882-887, September 2008.

[21] Y. G. Sun, L. Wang, and G. Xie, "Delay-dependent robust stability and stabilization for discrete-time switched systems with mode-dependent time-varying delays," Applied Mathematics and Computation, vol. 180, no. 2, pp. 428-435, 2006.

[22] Y. G. Sun, L. Wang, and G. Xie, "Delay-dependent robust stability and $H_{\infty}$ control for uncertain discrete-time switched systems with mode-dependent time delays," Applied Mathematics and Computation, vol. 187, no. 2, pp. 1228-1237, 2007.

[23] K. Hu and J. Yuan, "Improved robust $H_{\infty}$ filtering for uncertain discrete-time switched systems," IET Control Theory \& Applications, vol. 3, no. 3, pp. 315-324, 2009.

[24] D.-W. Ding and G.-H. Yang, " $H_{\infty}$ static output feedback control for discrete-time switched linear systems with average dwell time," IET Control Theory \& Applications, vol. 4, no. 3, pp. 381390, 2010.

[25] G. Zhai, B. Hu, K. Yasuda, and A. N. Michel, "Stability analysis of switched systems with stable and unstable subsystems: an average dwell time approach," in Proceedings of American Control Conference, pp. 200-204, June 2000.

[26] P. M. Igic, M. S. Towers, and P. A. Mawby, "A 2D physically based compact model for advanced power bipolar devices," Microelectronics Journal, vol. 35, no. 7, pp. 591-594, 2004.

[27] W. C. Lo, L. Wang, and B. Li, "Thermal transistor: heat flux switching and modulating," Journal of the Physical Society of Japan, vol. 77, no. 1, pp. 1-4, 2008.

[28] A. Benzaouia, A. Hmamed, and F. Tadeo, "Stability conditions for discrete 2D switching systems, based on a multiple Lyapunov function," in Proceedings of European Control Conference, pp. 23-26, 2009.

[29] A. Benzaouia, A. Hmamed, F. Tadeo, and A. El Hajjaji, "Stabilisation of discrete 2D time switching systems by state feedback control," International Journal of Systems Science, vol. 42, no. 3, pp. 479-487, 2011.

[30] Z. Xiang and S. Huang, "Stability analysis and stabilization of discrete-time 2D switched systems," Circuits, Systems, and Signal Processing, vol. 32, no. 1, pp. 401-414, 2013.

[31] L. Zhang and H. Gao, "Asynchronously switched control of switched linear systems with average dwell time," Automatica, vol. 46, no. 5, pp. 953-958, 2010.

[32] L. Zhang and P. Shi, "Stability, $l_{2}$-gain and asynchronous $H_{\infty}$ control of discrete-time switched systems with average dwell time," IEEE Transactions on Automatic Control, vol. 54, no. 9, pp. 2192-2199, 2009.

[33] Z. Xiang, C. Liang, and Q. Chen, "Robust $L_{2}-L_{\infty}$ filtering for switched systems under asynchronous switching," Communications in Nonlinear Science and Numerical Simulation, vol. 16, no. 8, pp. 3303-3318, 2011.

[34] Z. R. Xiang and R. H. Wang, "Robust stabilization of switched non-linear systems with time-varying delays under asynchronous switching," Proceedings of the Institution of Mechanical Engineers. Part I: Journal of Systems and Control Engineering, vol. 223, no. 8, pp. 1111-1128, 2009.

[35] Z. R. Xiang and R. H. Wang, "Robust control for uncertain switched non-linear systems with time delay under asynchronous switching," IET Control Theory \& Applications, vol. 3, no. 8, pp. 1041-1050, 2009. 
[36] Z. Xiang, Y.-N. Sun, and Q. Chen, "Robust reliable stabilization of uncertain switched neutral systems with delayed switching," Applied Mathematics and Computation, vol. 217, no. 23, pp. 9835-9844, 2011.

[37] S. Boyd, L. El Ghaoui, E. Feron, and V. Balakrishnan, Linear Matrix Inequalities in System and Control Theory, vol. 15 of SIAM Studies in Applied Mathematics, SIAM, Philadelphia, Pa, USA, 1994.

[38] L. Xie, "Output feedback $H_{\infty}$ control of systems with parameter uncertainty," International Journal of Control, vol. 63, no. 4, pp. 741-750, 1996. 


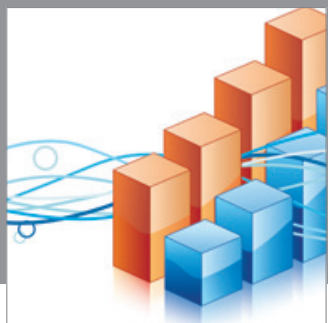

Advances in

Operations Research

mansans

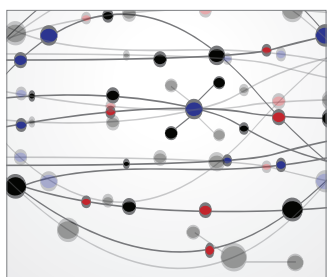

The Scientific World Journal
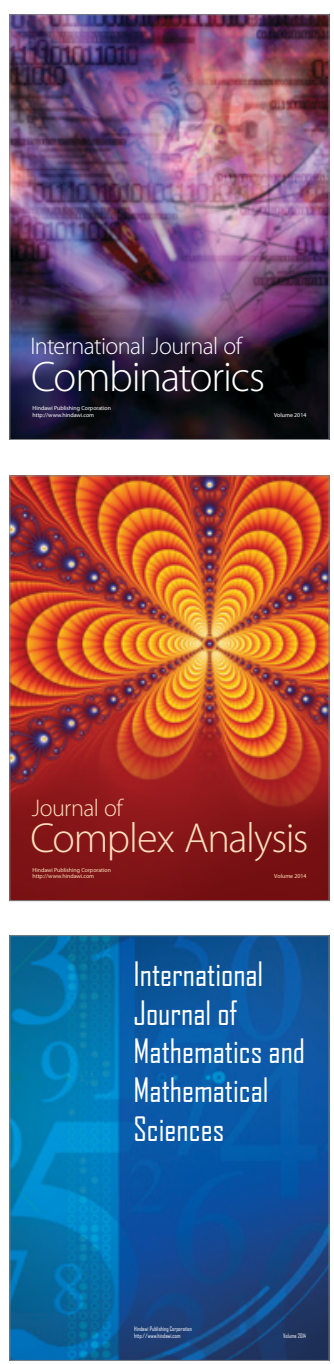
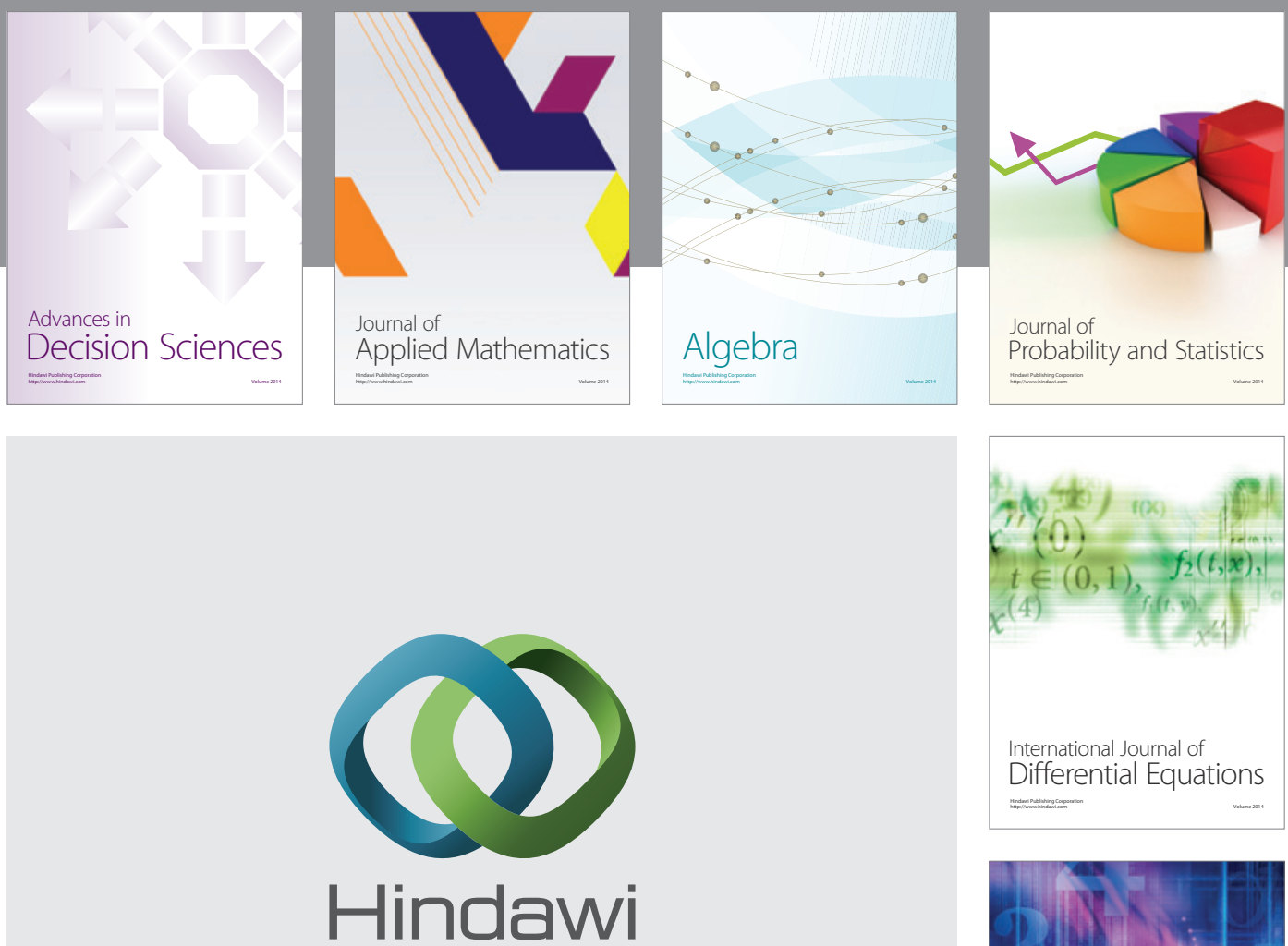

Submit your manuscripts at http://www.hindawi.com
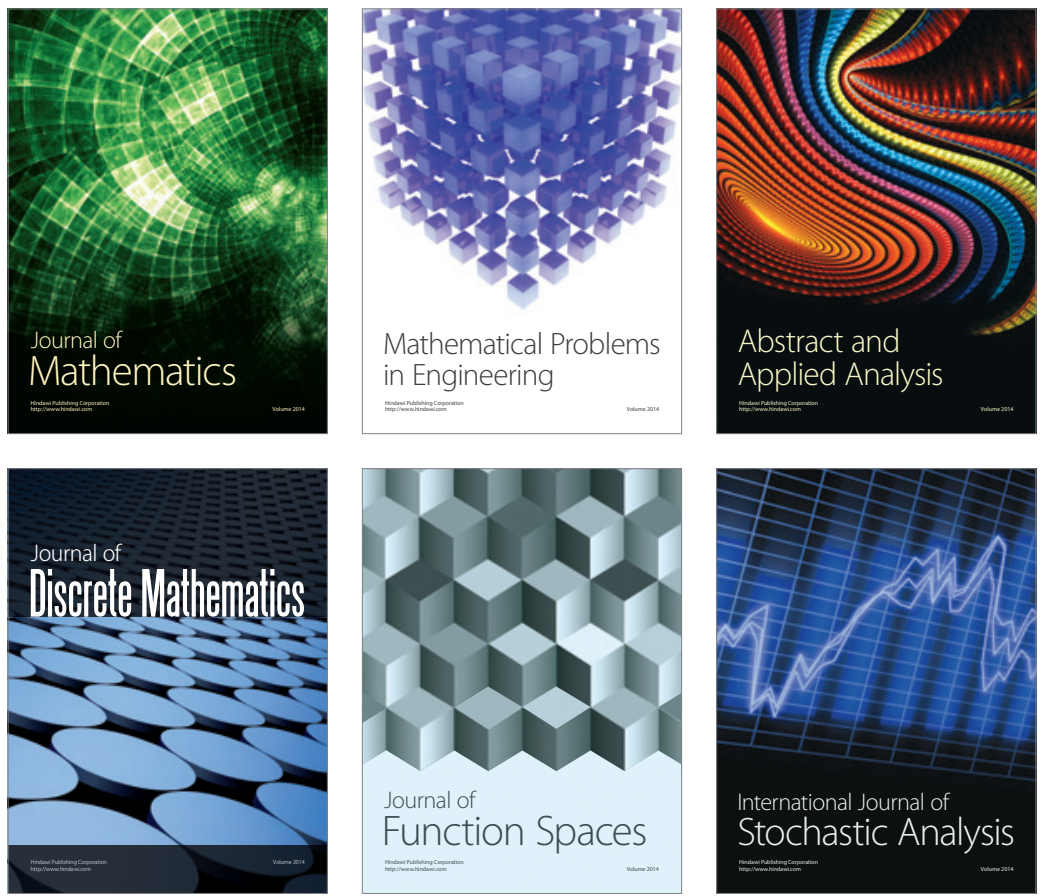

Journal of

Function Spaces

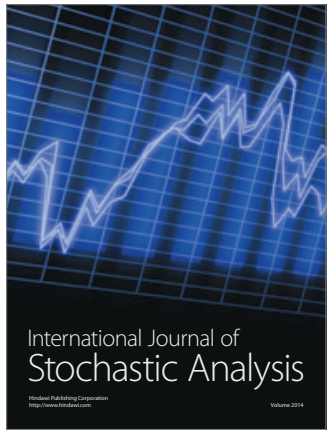

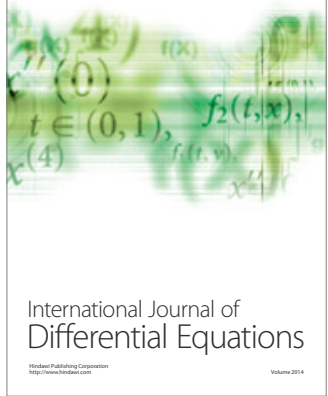
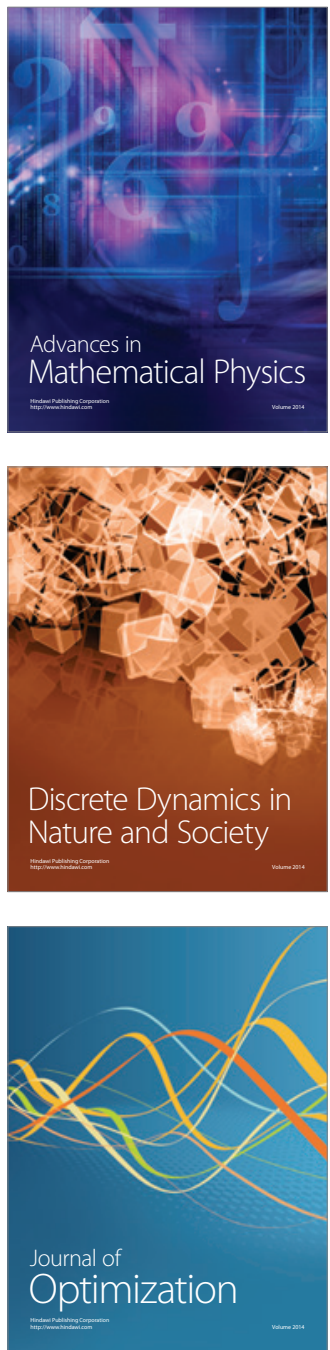